\title{
Brain Dynamics Underlying Training-Induced Improvement in Suppressing Inappropriate Action
}

\author{
Aurelie L. Manuel, ${ }^{1,2}$ Jeremy Grivel, ${ }^{2}$ Fosco Bernasconi, ${ }^{1,2}$ Micah M. Murray,,${ }^{1,3,4,5}$ and Lucas Spierer ${ }^{1}$ \\ ${ }^{1}$ Neuropsychology and Neurorehabilitation Service, ${ }^{2}$ Community Psychiatry Service, and ${ }^{3}$ Radiology Service, Vaudois University Hospital Center and \\ University of Lausanne, CH-1011 Lausanne, Switzerland, ${ }^{4}$ Electroencephalography Brain Mapping Core, Center for Biomedical Imaging, CH-1011 \\ Lausanne, Switzerland, and ${ }^{5}$ Department of Hearing and Speech Sciences, Vanderbilt University Medical Center, Nashville, Tennessee 37232
}

Inhibitory control, a core component of executive functions, refers to our ability to suppress intended or ongoing cognitive or motor processes. Mostly based on Go/NoGo paradigms, a considerable amount of literature reports that inhibitory control of responses to "NoGo" stimuli is mediated by top-down mechanisms manifesting $\sim 200 \mathrm{~ms}$ after stimulus onset within frontoparietal networks. However, whether inhibitory functions in humans can be trained and the supporting neurophysiological mechanisms remain unresolved. We addressed these issues by contrasting auditory evoked potentials (AEPs) to left-lateralized "Go" and right NoGo stimuli recorded at the beginning versus the end of $30 \mathrm{~min}$ of active auditory spatial Go/NoGo training, as well as during passive listening of the same stimuli before versus after the training session, generating two separate $2 \times 2$ withinsubject designs. Training improved Go/NoGo proficiency. Response times to Go stimuli decreased. During active training, AEPs to NoGo, but not Go, stimuli modulated topographically with training 61-104 ms after stimulus onset, indicative of changes in the underlying brain network. Source estimations revealed that this modulation followed from decreased activity within left parietal cortices, which in turn predicted the extent of behavioral improvement. During passive listening, in contrast, effects were limited to topographic modulations of AEPs in response to Go stimuli over the 31-81 ms interval, mediated by decreased right anterior temporoparietal activity. We discuss our results in terms of the development of an automatic and bottom-up form of inhibitory control with training and a differential effect of Go/NoGo training during active executive control versus passive listening conditions.

\section{Introduction}

Inhibitory control is the ability to suppress intended or ongoing cognitive or motor processes and allows flexible adaptation to changing environmental contingencies (Aron et al., 2004, 2007). Investigations of inhibitory control principally rely on $\mathrm{Go} / \mathrm{NoGo}$ paradigms requiring speeded responses to one class of stimuli ("Go") while withholding responses to another class of stimuli ("NoGo"). Convergent evidence indicates that the suppression of prepotent responses to NoGo and the monitoring of conflicts between divergent response requirements to Go and NoGo are prominently controlled by top-down mechanisms. Neuroimaging and lesion data report that inhibitory control involves brain regions traditionally associated with higher-order executive functions, including the inferior frontal cortex and temporoparietal areas

\footnotetext{
Received April 22, 2010; revised Aug. 9, 2010; accepted Aug. 13, 2010.

This work was supported by an interdisciplinary grant from the Faculty of Biology and Medicine, University of Lausanne (L.S., J.G.) and a grant from the Pierre Mercier Foundation for Science (L.S., J.G.). M.M.M. is supported by Swiss National Science Foundation Grant 3100A0-118419/1. Cartool software has been programmed by Denis Brunet (Functional Brain Mapping Laboratory, Geneva, Switzerland) and supported by the Center for Biomedical Imaging of Geneva and Lausanne. We thank Gilles Pourtois and Roland Vocat for their help in programming the paradigm.

Correspondence should be addressed to Dr. Lucas Spierer, Faculty of Biology and Medicine, Université de Lausanne, Neuropsychology and Neurorehabilitation Service, Centre Hospitalier Universitaire Vaudois, Avenue PierreDecker 5, CH-1011, Lausanne, Switzerland. E-mail: Lucas.Spierer@chuv.ch.

DOI:10.1523/JNEUROSCI.2064-10.2010

Copyright $\odot 2010$ the authors $\quad 0270-6474 / 10 / 3013670-09 \$ 15.00 / 0$
}

(Rubia et al., 2001; Aron et al., 2004; Polich, 2007). Further corroborating the role of higher-order cognitive processes, event-related potential (ERP) studies of Go/NoGo tasks demonstrated that the suppression of prepotent responses manifests over processing stages subsequent to initial sensory functions at latencies of $150-400 \mathrm{~ms}$ after stimulus onset (NoGo-N2/P3 components peaking over frontocentral electrodes) (Pfefferbaum et al., 1985; Jodo and Kayama, 1992; Eimer, 1993; Schröger, 1993; Falkenstein et al., 1995, 1999; Kiefer et al., 1998; Kaiser et al., 2006).

Whether inhibitory control can be trained and the supporting neural mechanisms remain unresolved. Two alternative, nonexclusive hypotheses can be drawn about this issue. First, based on the compelling evidence that $\mathrm{Go} / \mathrm{NoGo}$ proficiency relies on the engagement of top-down executive control (Aron et al., 2004; Dillon and Pizzagalli, 2007), one could hypothesize that improvement of inhibitory control would be solely supported by the reinforcement of these top-down processes. In line with this assumption, one ERP study demonstrated that Go/NoGo practice modulates responses to both Go and NoGo stimuli at 160-240 $\mathrm{ms}$ and to NoGo only at 240-320 ms (Schapkin et al., 2007). The authors interpreted this pattern of results in terms of changes in higher-order processes involving the comparison of the stimuli with a memory template and the subsequent inhibition of responses to NoGo stimuli.

Alternatively, some evidence for modality-dependent NoGo inhibition (e.g., inhibition-related activity within supratemporal 


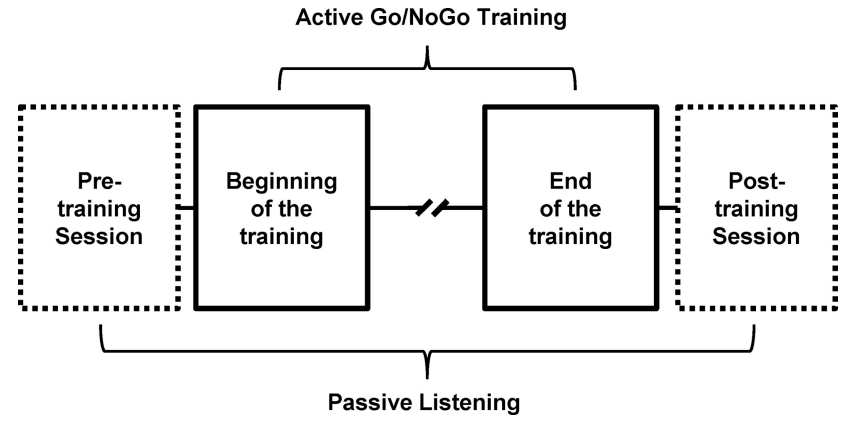

Figure 1. Experimental design. Each participant completed one training session (active Go/ NoGo training) as well as two sessions of passive listening before and immediately after the training sessions (passive pretraining and posttraining sessions).

plane in auditory but not visual Go/NoGo) (Walther et al., 2010) suggested a role for bottom-up and lower-order sensorycognitive processes in Go/NoGo proficiency, challenging the view that inhibitory control involves solely top-down inputs from higher-order executive modules. In support, recent psychophysical models advanced that fast, feedforward, and automatic forms of inhibitory control develop with Go/NoGo practice that are driven by repeated associations between NoGo stimuli and response withholding (Shiffrin and Schneider, 1977; Logan, 1988; Verbruggen and Logan, 2008).

To identify the spatiotemporal brain mechanisms underlying training-induced plasticity in inhibitory control, we contrasted electrical neuroimaging analyses of auditory evoked potentials (AEPs) to Go and NoGo stimuli at the beginning versus the end of an auditory spatial Go/NoGo training. These analyses differentiate effects attributable to topographic modulations from those attributable to changes in response gain, allowing for a better description of likely neurophysiologic mechanisms. To test whether the training impacted preattentive, task-independent stimulus representation rather than solely top-down executive control, we further investigated how training modified AEPs to the trained stimuli presented during passive listening.

\section{Materials and Methods}

Participants. Eleven healthy volunteers participated in the study (all male, all right-handed using the Edinburgh questionnaire) (Oldfield, 1971), aged $22-39$ years (mean $\pm S D, 29.36 \pm 1.56$ years). Each participant provided written, informed consent to participate in the study. No participant had a history of neurological or psychiatric illness, and all reported normal hearing. All procedures were approved by the Ethics Committee of the Faculty of Biology and Medicine of the Vaudois University Hospital Center and University of Lausanne.

Stimuli. Auditory stimuli were $150 \mathrm{~ms}$ noise bursts (200-500 Hz bandpass filtered; $5 \mathrm{~ms}$ rise/fall; $44.1 \mathrm{kHz}$ sampling; generated using Adobe Audition 1.0; Adobe Systems), lateralized by means of a right- or left-ear leading interaural time difference of $770 \mu \mathrm{s}$, which led to perceived lateralization of $\sim 80^{\circ}$ from the central midline (Blauert, 1997). The sounds were presented via insert earphones (ER-4P; Etymotic Research) at a level judged comfortable by the participant $(\sim 85 \mathrm{~dB}$ sound pressure level, measured using a CESVA SC-160 sound pressure meter).

Procedure and task. Each participant completed one training session that we refer to as "active Go/NoGo training," as well as two sessions of passive listening before and immediately after the training sessions (Fig. 1). Throughout the experiment, participants were seated in an electrically shielded and sound-attenuated booth in front of a 19 inch liquid crystal display screen. Stimulus delivery and response recording were controlled using E-prime 2.0.

Active Go/NoGo training. The training session consisted in an auditory spatial Go/NoGo task in which participants had to respond as quickly as possible using the forefinger of the right hand via a manual response-box button to left-lateralized sounds (Go stimuli, hereafter termed LG) and to withhold responses to right-lateralized sounds (NoGo stimuli, termed RNG). The stimulus-response mapping was straightforward, and the LG and RNG sounds were easily discriminated. Respectively, these features minimize confounding effects of either learning the rules of the task or learning to discriminate between the spatial positions of the sounds on the Go/NoGo performance improvement. Each trial started with the presentation of a visual cue (centrally presented gray cross on a black background) of a randomly determined duration ranging from 1000 to $1900 \mathrm{~ms}$. At the same time that the cross was turned off, the LG and RNG sounds were presented and response was recorded.

In the Go conditions, a feedback was provided immediately after the response (see below). To avoid that any differences between ERPs to LG and RNG trials followed from differences in relative novelty or presentation frequency, they were presented with an equal probability of 0.5 . This balance further ensured that, during the passive listening portion, pretraining versus posttraining difference in EEG responses to LG and RNG stimuli were not attributable to differences in the number of intervening stimulus presentations.

The active Go/NoGo training was divided into three experimental sessions. Each session started with a calibration block of 16 randomly presented trials (eight LG and eight RNG), followed by two test blocks each of 80 randomly presented trials (40 LG and 40 RNG). The calibration blocks were used to individually adjust the task difficulty and to maintain time pressure across the whole experiment. This was accomplished in the following way. During each calibration phase, the mean response time (RT) to LG trials was calculated online and used to determine the individual participant's RT threshold (RTt), which was set slightly below current response speed (i.e., calculated as $80 \%$ of the mean RT from the calibration block). During the test block, a Go response RT was considered as correct if it was below the $80 \%$ RTt of the immediately preceding calibration phase. Otherwise, a feedback screen indicating "too late!" was displayed immediately after the Go response (slow hit). On each trial, feedback on global accuracy was displayed (mean percentage of correct trials, including fast hit and correct rejection). Participants were not informed about this thresholding procedure. Except the global accuracy, no visual feedback was displayed after fast hits or false alarms (FAs) (i.e., a response to a NoGo stimulus) (for a similar procedure, see Vocat et al., 2008). The whole Go/NoGo training session included a total of 528 stimuli [ 160 stimuli in the test block +16 stimuli in the calibration block) $\times 3$ sessions $=528$ stimulus $]$ and lasted for a total of $\sim 35$ min. After the completion of each session, a rest period of 10 min was provided to participants.

Passive pretraining and posttraining sessions. Pretraining and posttraining passive sessions consisted of six blocks of passive listening. In each block, the stimuli were presented in a pseudorandom order with a random interstimulus interval (ISI) ranging from 700 to $900 \mathrm{~ms}$. The ISIs were reduced in the passive listening part of the study compared with the active Go/NoGo training session in which the ISI ranged from 1000 to $1900 \mathrm{~ms}$. ISIs were reduced to increase the number of presented stimuli (and thereby the signal-to-noise ratio) while keeping the experiment as short as possible for participants. A corresponding reduction of ISI was not applicable in the active task, because the presentation of the cue, the recording of participant's response, and the presentation of the feedback were included between the presentations of the two stimuli. We would note that in the differences in ISIs between active and passive conditions could constitute a potential confounds when comparing the results of the two tasks. The blocks were randomized across pretraining and posttraining sessions and across subjects. In each block, the same LG and RNG stimuli as in the active Go/NoGo training were presented (69 LG and 69 RNG per block). Seven other additional sounds were presented in the framework of another experiment focusing on the generalization of the effects of the Go/NoGo training and were not analyzed in the present study. Each participant completed three blocks before and three blocks after the training tasks while watching a muted film; they were instructed to ignore the auditory stimuli. The pretraining and posttraining session lasted for $\sim 30$ min each. 
EEG acquisition and preprocessing. Continuous EEG was acquired at $1024 \mathrm{~Hz}$ though a 128-channel Biosemi ActiveTwo system referenced to the common mode sense/driven right leg ground (which functions as a feedback loop driving the average potential across the montage as close as possible to the amplifier zero). Before group averaging, data at artifact electrodes from each participant were interpolated (Perrin et al., 1987). EEG epochs from $100 \mathrm{~ms}$ before to $452 \mathrm{~ms}$ after stimulus onset (i.e., 102 data points before and 463 data points after stimulus onset) were averaged, for each participant, for LG and RNG trials from the two first ("beginning" condition) and two last ("end" condition) blocks of the active training session and from the three pretraining and three posttraining sessions during passive, generating separate $2 \times 2$ within-subject designs for the active training and passive listening portions of the experiment with factors of section (beginning and end of the active training; "pre" and "post" training in the case of the passive listening portion) and stimulus (LG and RNG). In addition to a $\pm 80 \mu \mathrm{V}$ artifact rejection criterion, EEG epochs containing eye blinks or other noise transients were removed after visual inspection. Data were baseline corrected using the $100 \mathrm{~ms}$ prestimulus period, bandpass filtered $(0.18-40 \mathrm{~Hz})$, and recalculated against the average reference.

During active training, the average \pm SEM number of accepted epochs was $75 \pm 2$ for the beginning LG, $72 \pm 3$ for the beginning RNG, $71 \pm 5$ for the end LG, and $62 \pm 5$ for the end RNG conditions. A $2 \times 2$ repeatedmeasures ANOVA with factors of section and stimulus (as performed for the ERP analyses) revealed a main effect of section $\left(F_{(1,10)}=5.72, p=0.03\right)$. Neither the main effect of stimulus nor the interaction term were significant.

During passive listening, the average \pm SEM number of accepted epochs was $170 \pm 8$ for the pre LG, $170 \pm 9$ for the pre RNG, $182 \pm 6$ for the post LG, and $180 \pm 8$ for the post RNG conditions. These values did not statistically differ.

EEG analyses and source estimations. Because of the leftward shift of RTs with training and the fact that participants responded to Go but not NoGo stimuli, confounding stimulus $\times$ section interactions could have occurred as a result of difference in the latency of the activity related to response button press. Therefore, in the analyses of ERPs from active training, we considered only effects occurring over a window limited to the first $100 \mathrm{~ms}$ after stimulus onset, i.e., before the minimal latency of response initiation in the motor cortex occurring $\sim 100 \mathrm{~ms}$ before the execution of the button press (the shortest mean RTs measured in our study were $\sim 200 \mathrm{~ms}$ ) (Thorpe and Fabre-Thorpe, 2001). Consequently, the N2/P3 complex occurring 150-400 ms after onset will not be analyzed here. Plus, because of the large psychophysical distance and the equal probability of presentation between the Go and NoGo stimuli in our study, large N2/P3 responses would not be expected (Nieuwenhuis et al., 2003, 2004). In our ERP analyses, we do not provide details on effects of block nor main effect of stimuli because they can be attributed to, respectively, unspecific effects of stimulus repetition and psychophysical differences between left-lateralized Go and right-lateralized NoGo stimuli, both effects being outside the scope of the present study. Main effects of stimulus could also be explained in terms of spatial attention, because Go stimuli were always left-lateralized. Thus, any differences could reflect participants' ability to deploy their attention rather than to response inhibition per se. Such effects of attention have been observed as modulations of the N2/P3 responses (i.e., after $\sim 200 \mathrm{~ms}$ after stimulus onset) (Schröger, 1993). As will be clear below in Results, however, the present effects occur within the initial $100 \mathrm{~ms}$ after stimulus onset and follow from interactions between stimulus type and block.

Topographic analyses (implemented in Cartool software developed by D. Brunet, Functional Brain Mapping Laboratory, Geneva, Switzerland) were performed to determine whether the configuration of intracranial generators changed across either or both factors (i.e., section and stimulus). These methods have been detailed previously and have many analytical and interpretational benefits over canonical AEP waveform analyses (Murray et al., 2008). We provide only the essentials here. Major impetuses for the use of the present analyses were the ability to circumvent interpretational issues attributable to the reference-dependent nature of AEPs and to differentiate effects arising from topographic modulations from effects resulting from changes in response strength. Moreover, the analyses used here require minimal experimenter selec- tion of either the electrodes or time periods of interest, which are two major sources of potential bias in AEP investigations.

Hierarchical clustering based on an atomize and agglomerate approach was performed to identify the pattern of predominating topographies (maps) in the cumulative group-averaged data (Murray et al., 2008). In this approach, the number of clusters initially equals the number of data points in the concatenated group-averaged dataset (i.e., 565 in the present study). This number is then sequentially reduced by identifying the cluster with the lowest global explained variance (GEV) with respect to all other clusters (for a recent publication of formulae, see Murray et al., 2008). The data from this cluster are then reassigned to one of the surviving clusters. The optimal number of clusters to describe the dataset is identified using a modified Krzanowski-Lai criterion (Tibshirani et al., 2005) (for an approach based on a modified crossvalidation criterion, see Pascual-Marqui et al., 1995). These steps are all a hypothesis generation tool that is then statistically evaluated using singlesubject data. Differences in the pattern of maps observed between conditions in the group-averaged data were tested by calculating the spatial correlation between these "template" maps from the group-averaged data and each time point of single-subject data from each experimental condition (referred to as "fitting"). For each participant, we calculated the GEV of each template map within the single-subject AEPs. In colloquial terms, GEV can be understood as the average (over the fitted time period) spatial correlation between a given template map and an individual's data from a specific condition that is weighted by the global field power at each time point over the averaged time period. In this way, GEV provides a measure across participants of how well a given template map accounts for a given condition over a specific time period.

We estimated the sources in the brain using a distributed linear inverse solution and the local autoregressive average (LAURA) regularization approach (Grave de Peralta Menendez et al., 2001; Grave-de Peralta et al., 2004) (for a comparison of inverse solution methods, see Michel et al., 2004). LAURA selects the source configuration that better mimics the biophysical behavior of electric fields (i.e., activity at one point depends on the activity at neighboring points according to electromagnetic laws). Homogenous regression coefficients in all directions and within the whole solution space were used. The solution space is based on a realistic head model and included 3005 nodes selected homogeneously distributed within the gray matter of the average brain of the Montreal Neurological Institute (courtesy of R. Grave de Peralta Menendez and S. Gonzalez Andino, University Hospital of Geneva, Geneva, Switzerland). The results of the above topographic pattern analysis defined time periods of stable topography for which intracranial sources were estimated and statistically compared at each node level between conditions using the same section $\times$ stimulus within-subject design as in the topographic pattern analysis. A spatial criterion of minimum 36 contiguous points was applied in the statistical parametric mapping procedure.

\section{Results}

\section{Behavioral results}

Participants completed a $35 \mathrm{~min}$ Go/NoGo training session during which they were instructed to respond as quickly as possible to left-lateralized sounds (LG stimuli) while withholding responses to right-lateralized sounds (RNG stimuli). We indexed behavioral performance by RTs to Go stimuli, the percentage of hits (responded Go stimuli) and FAs (NoGo errors). As for the EEG analyses, behavioral data were separately averaged for the beginning and end conditions (i.e., two first and two last blocks of the training session, respectively). RTs significantly decreased with training (mean \pm SEM, beginning, $274 \pm 28 \mathrm{~ms}$; end, $228 \pm$ $15 \mathrm{~ms} ; t_{(10)}=4.673 ; p<0.001$ ). Because participants were required to respond with their right hand to the left-lateralized Go stimuli, the speeding of response time may be partly attributable to a reduction of the Simon effect with training (Proctor and Shao, 2010). The mean percentage of hits was at ceiling from the beginning of the training and did not modulate across sections of the training session (beginning, $98.9 \pm 0.3 \%$; end, $98.5 \pm 0.4 \%$; 
$\left.t_{(10)}=0.69 ; p=0.50\right)$. The mean percentage of FAs significantly increased with training (FA beginning, $7.5 \pm 0.8 \%$; FA end, $\left.14.3 \pm 1.1 \% ; t_{(10)}=-3.90 ; p<0.001\right)$. However, we are reluctant to interpret the increase in commission errors as reflecting a decrease in Go/NoGo proficiency. Because of the implementation of the auto-adaptative thresholding of the minimal RTs at which a response was reported as correct, a strong time pressure was maintained constantly across the training session, even when participants had reached their maximal response speed (i.e., $\sim 200 \mathrm{~ms}$ ). At the end of the training, participants were unable to continue accelerating their responses to Go stimuli. As a result, they committed more FAs (for discussion on this issue, see Falkenstein et al., 2000). Further arguing against the same training-induced mechanisms for decrease in RT and the increase in FA with training, speed-accuracy tradeoff analyses revealed no relation between the modulation in RT and in FA with training $\left(r_{(9)}=0.14 ; p=0.67\right)$.

\section{Electrical neuroimaging results}

Active Go/NoGo training session

Hierarchical clustering was performed on the AEPs to identify the pattern of predominating topographies (maps) of the electric field at the scalp in the cumulative group-averaged data. The output of the topographic pattern analysis of the collective data during active training is displayed in Figure $2 a$ [see also AEP waveforms at a vertex electrode $(\mathrm{Cz})]$. The GEV of the results of the cluster analysis was $96.96 \%$. This topographic pattern analysis identified the same sequence of stable maps for trials from the beginning and end conditions and LG and RNG trial types, with the exception of the 61-104 ms after stimulus. Over this time period, these maps were differentially observed across sections and stimuli.

Using the single-subject data from each condition, the GEV of each of the maps identified over period of topographic modulation in the group-averaged AEPs was then calculated to obtain a quantitative estimate of how well they accounted for individual participants' AEPs over the same time interval (Fig. 2b).

There was a significant interaction between section, stimulus, and map $\left(F_{(1,10)}=5.672, p=0.039\right)$. Follow-up ANOVAs were therefore conducted for each stimulus separately. There was a significant interaction between section and map for the RNG but not LG stimuli as a function of training section (section $\times$ map interaction; $\mathrm{LG}, F_{(1,10)}=0.087, p=0.774 ; \mathrm{RNG}, F_{(1,10)}=11.27$, $p=0.007)$. Over the same time period, there was a significant interaction between section and map $\left(F_{(1,10)}=6.263, p=0.031\right)$, indicating an unspecific effect of training on topographic responses to both RNG and LG stimuli. LAURA distributed source estimations were calculated over the 61-104 ms poststimulus period (Fig. $2 c$ ), i.e., when the topographic pattern analysis showed significant interaction between factors section, stimulus, and map. To do so, AEPs for each participant and each experimental condition separately were first averaged across the above mentioned time period to generate one data point per participant and experimental condition. Source estimations were then calculated, and the scalar value of each solution point was submitted to a $2 \times 2$ repeated-measures ANOVA with section and stimulus as within-subject factors. There was a significant main effect of section over right temporoparietal areas $\left(F_{(1,10)}>4.965, p<0.05\right)$. The main effect of stimuli included frontoparietal regions bilaterally $\left(F_{(1,10)}>4.965, p<0.05\right)$. This is consistent with models of auditory spatial processing and spatial attention that implicate frontoparietal circuits (Spierer et al., 2007, 2008, 2010). The fact that both frontal and parietal regions were synchronously ob- served over this time window is suggestive of a degree of parallel processing. However, we cannot exclude the possibility of directionality at a finer temporal scale, because data were first averaged in time across the 61-104 ms period.

Of particular relevance to the goals of the present study, there was a significant interaction between these factors in a left temporoparietal cluster $\left(F_{(1,10)}>4.965, p<0.05\right)$ (Fig. $2 d$ ). Follow-up statistical tests were performed to determine the basis for this interaction. The scalar values of the solution points comprised within the region of interest (ROI) showing the section $\times$ stimuli interaction were extracted and averaged for each subject and condition. The resulting values were compared across sections for LG and RNG stimuli separately. These analyses revealed a significant decrease in the activation strength of the temporoparietal ROI between beginning and end conditions for the RNG, but not for LG, stimuli $\left(t_{(10)}=4.621, p<0.01\right.$ and $t_{(10)}=1.045$, $p=0.321$, respectively) (Fig. $2 e$ ).

Correlational analysis performed between scalar values of the ROI showing the section $X$ stimulus interaction and behavioral performance revealed that training-induced modulations in response strength to RNG within the temporoparietal ROI (beginning-end) negatively correlated with modulations in RT (beginning-end; solution point showing the maximal correlation within the ROI, $r_{(9)}=-0.67 ; p<0.03$ ) (Fig. $2 f$ ). The more activity decreased within this region across training blocks, the more the speed of performance improved.

\section{Passive pretraining and posttraining session}

The topographic pattern analysis identified the same sequence of stable maps for trials from the pretraining and posttraining conditions and LG and RNG trial type, with the exception of two poststimulus intervals. The output of the topographic pattern analysis of the collective data from the passive listening portions is displayed in Figure $3 a$ [see also AEP waveforms at an exemplar electrode $(\mathrm{Cz})]$. The GEV of the results of the cluster analysis was $90.87 \%$.

Two maps were identified in the group-averaged data over the 31-81 ms interval, and these were differentially observed across sections and stimuli. There was a significant interaction between factors section, stimulus, and map over the 31-81 ms poststimulus period $\left(F_{(1,10)}=9.003, p=0.013\right)$. Follow-up ANOVAs were therefore conducted for each stimulus separately. There was a significant interaction between section and map for the LG but not RNG stimuli as a function of training section (section $\times$ map interaction; $\mathrm{LG}, F_{(1,10)}=6.145, p=0.033 ; \mathrm{RNG}, F_{(1,10)}=0.609$, $p=0.453)$. This pattern of results indicates that the intervening training session modulated topographically responses to passively presented LG but not RNG stimuli (Fig. $3 b$ ). Over the 248-350 ms interval, there was a significant interaction between stimulus and map $\left(F_{(1,10)}=5.264, p=0.045\right)$, indicating that distinct topographic patterns accounted for responses to the two stimuli. This differential processing of the two stimuli likely followed from the psychophysical differences between the left- and right-lateralized sounds (for corresponding findings, see Schröger, 1993; Spierer et al., 2007).

LAURA distributed source estimations were calculated over the 31-81 ms poststimulus period (Fig. 3c), i.e., when the topographic pattern analysis showed a significant interaction between factors of section, stimulus, and map. There was a significant interaction between section and stimulus in a cluster including anterior parietal and superior temporal regions $\left(F_{(1,10)}>4.965\right.$, $p<0.05$ ) (Fig. $3 d$ ) but no main effect of stimuli or section. Follow-up statistical tests were performed to better understand 
a Temporal segmentation of AEPs

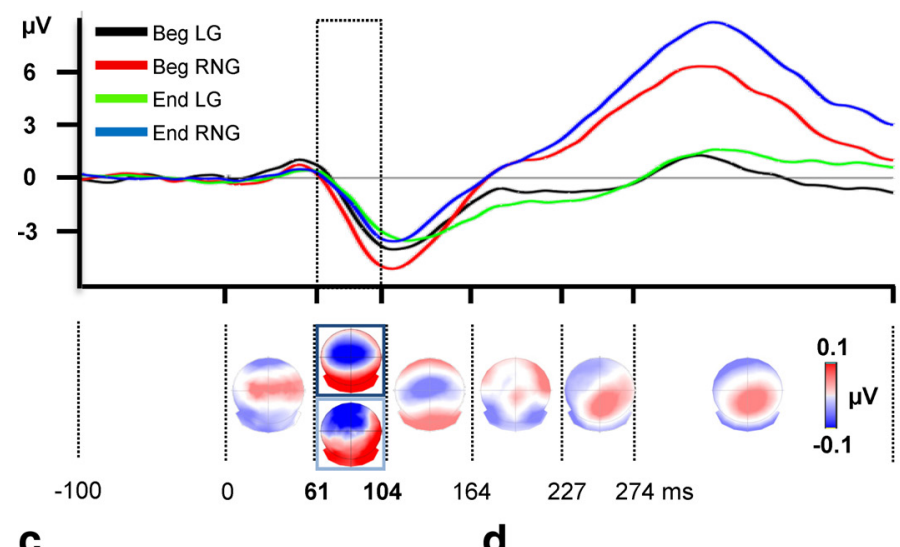

C

Group-Averaged LAURA

Sources Estimation (61-104 ms)

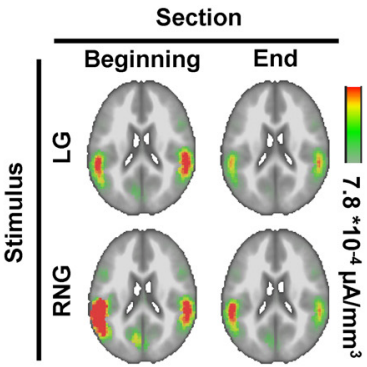

Node-Wise Section*Stimulus Interaction (61-104 ms)

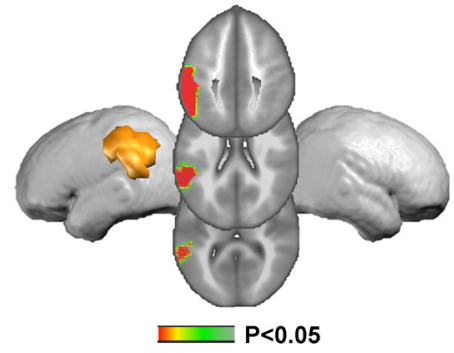

b Individual Subject Fitting (61-104ms)

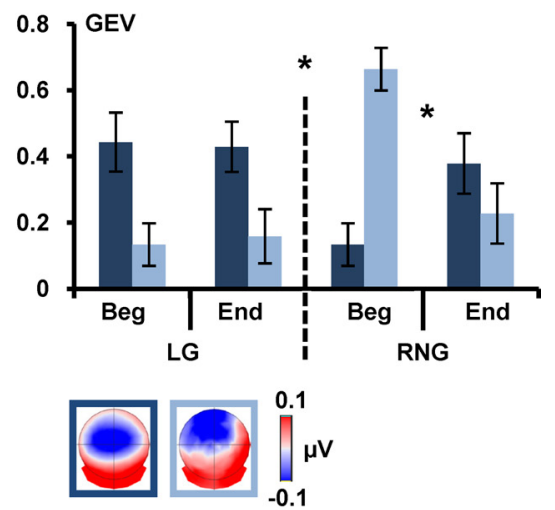

e

Left temporo-parietal ROI Activity (61-104 ms)

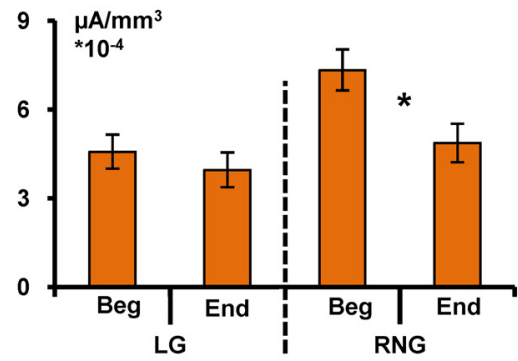

f Correlation between change in Go/NoGo performance and in responses to RNG
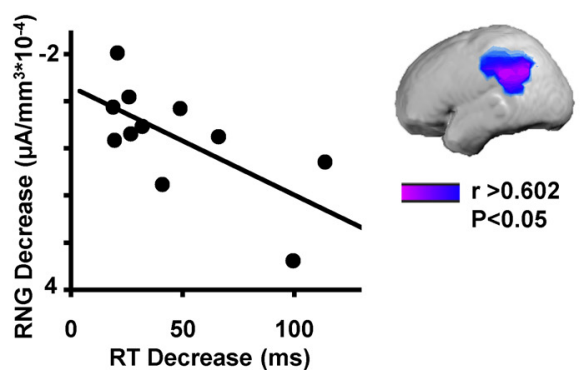

Figure 2. Active Go/NoGo training: electrical neuroimaging results. $\boldsymbol{a}$, The AEP in response to the beginning for Go (LG, black trace) and NoGo (RNG, red) and end for Go (green) and NoGo (blue) of the experiment are displayed in microvolts as a function of time for the Cz electrode. Topographic pattern analyses identified six time periods of stable electric field topography across the collective $452 \mathrm{~ms}$ poststimulus period. All topographies (i.e., maps) are shown with the nasion upward and left scalp leftward. For one of these time periods (61-104 ms), multiple maps were identified in the group-averaged AEPs. These maps are framed in blue. $\boldsymbol{b}$, The reliability of this observation at the group-averaged level was then assessed at the single-subject level using a spatial correlation fitting procedure. The GEV of each template map provides a measure across subjects of how well a given template map accounts for a given condition over the 61-104 ms time period (see Materials and Methods). Over the 61-104 ms period after stimulus, different maps (framed in dark and light blue) described AEPs in response to the Go and NoGo stimuli as a function of training (beginning/end). There was a significant three-way interaction between section, stimulus, and map. Error bars indicate SEM. c, Group-averaged distributed linear source estimations were calculated over the 61-104 ms poststimulus period for each experimental condition (scale indicated), when AEPs analyses revealed a significant topographic modulation across conditions. $\boldsymbol{d}$, Node-wise section $\times$ stimulus ANOVA on source estimation over the 61-104 ms interval revealed significant section $\times$ stimuli interactions within a left temporoparietal cluster. $\boldsymbol{e}$, Follow-up analyses on the mean scalar value of the ROI revealed a decrease in the left temporoparietal cortex for the NoGo stimuli as a function of training. $f$, Node-wise correlations between response time to Go stimuli and the activity within the cluster showing the significant section $\times$ stimulus interaction revealed that the more performance improved, the more response strength to NoGo stimuli decreased within the left temporoparietal ROI.

the basis of the interaction, revealing a significant decrease in the activation strength of the right anterior temporoparietal ROI between pretraining and posttraining conditions for the LG but not RNG stimuli $\left(t_{(10)}=3.100, p<0.02\right.$ and $t_{(10)}=-0.337, p=$ 0.743 , respectively) (Fig. $3 e$ ).

\section{Discussion}

We identified the spatiotemporal brain dynamics underlying training-induced plasticity in inhibitory control. The processes engaged for responding to Go stimuli while withholding re- sponses to NoGo were subject to facilitation; RTs significantly decreased to Go stimuli during the course of active training. Brain mechanisms associated with such plasticity in inhibitory control were first identified by applying electrical neuroimaging analyses to AEPs in response to Go and NoGo stimuli recorded at the beginning versus the end of 35 min active auditory spatial Go/NoGo training. Then, we applied the same contrast to EEG responses recorded during passive listening of the Go and NoGo stimuli immediately before and after the active training session. The collective findings support a model wherein learning- 
a

Temporal segmentation of AEPs

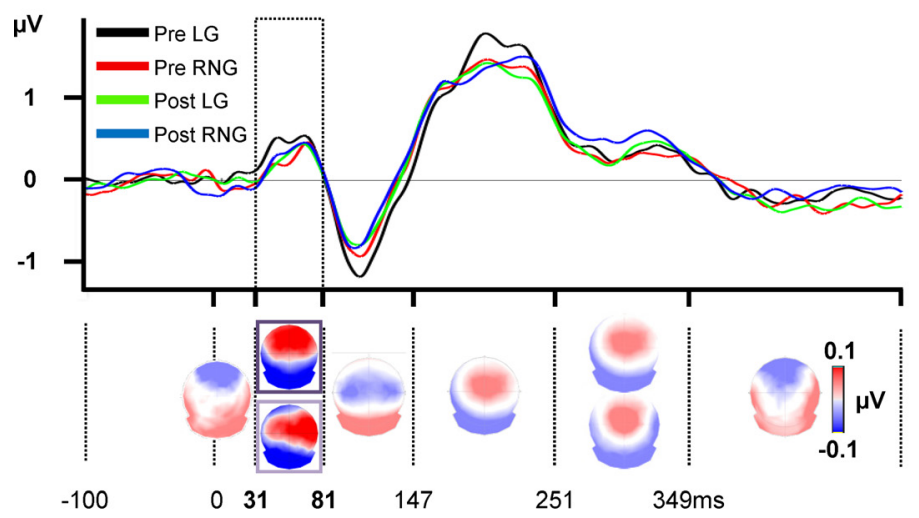

C
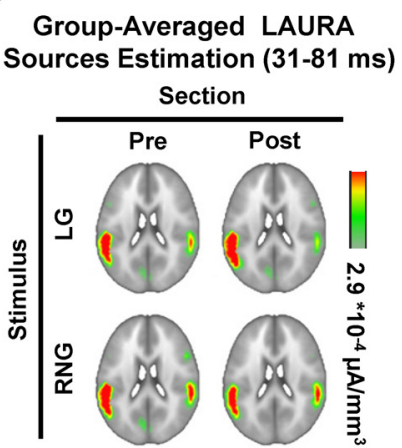

d
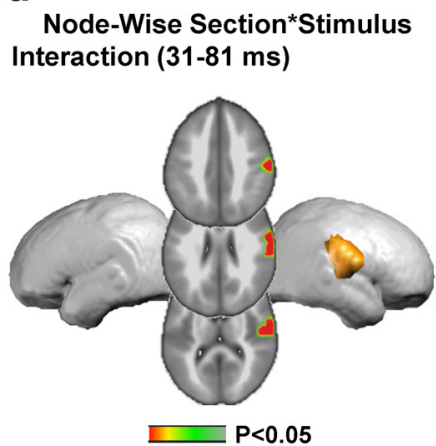

b
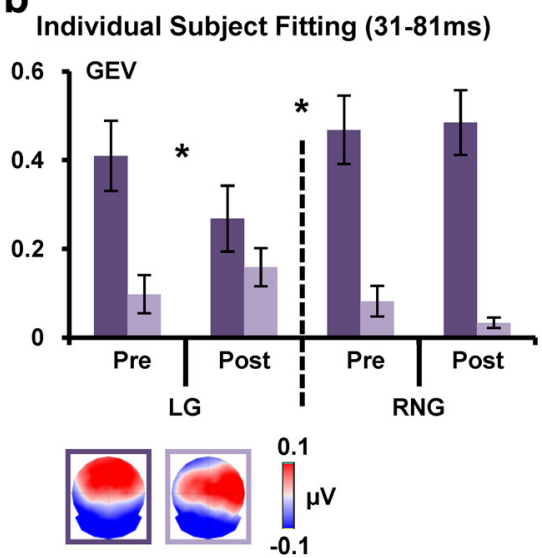

e

Right temporo-parietal ROI Activity (31-81 ms)

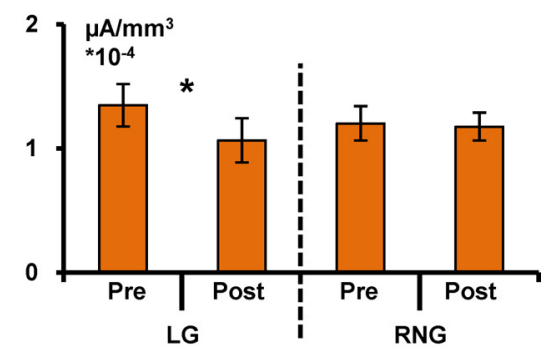

Figure 3. Pretraining and posttraining passive listening: electrical neuroimaging results. $\boldsymbol{a}$, The AEP in response to the pretraining Go (LG, black trace) and NoGo (RNG, red) and posttraining Go (green) and NoGo (blue) stimuli are displayed in microvolts as a function of time for the Cz electrode. Topographic pattern analyses identified six time periods of stable electric field topography across the collective 452 ms poststimulus period. All topographies (i.e., maps) are shown with the nasion upward and left scalp leftward. For the 31-81 ms period, multiple maps were identified in the group-averaged AEPs. These maps are framed in purple. $\boldsymbol{b}$, The reliability of this observation at the group-averaged level was then assessed at the single-subject level using a spatial correlation fitting procedure. The GEV of each template map provides a measure across subjects of how well a given template map accounts for a given condition over the 61-104 ms time period (see Materials and Methods). Over the 31-81 ms period after stimulus, different maps (framed in dark and light purple) described AEPs in response to the $\mathrm{Go}$ and NoGo stimuli as a function of training (pre/post). There was a significant three-way interaction between section, stimulus, and map. Error bars indicate SEM. c, Group-averaged distributed linear source estimations were calculated over the 31-81 ms poststimulus period for each experimental condition (scale indicated), when AEPs analyses revealed a significant topographic modulation across conditions. $\boldsymbol{d}$, Node-wise section $\times$ stimulus ANOVA on source estimation over the 31-81 ms interval revealed significant section $\times$ stimuli interactions within a right temporoparietal cluster. $\boldsymbol{e}$, Follow-up analyses on the mean scalar value of the ROI revealed a decrease in the right temporoparietal cortex for the Go stimuli as a function of training.

induced plasticity in inhibitory control manifests during early, low-level stages, affects prepotent responses to stimuli, and extends beyond training to impact passive listening of previously behaviorally relevant stimuli.

Responses to NoGo but not Go stimuli during active training modulated the AEP topography over the 61-104 ms poststimulus onset interval, indicative of the engagement of distinct configurations of intracranial generators. Source estimations revealed that this modulation followed from decreased activity within left temporoparietal cortices in response to NoGo stimuli that in turn correlated with the extent of RT facilitation to Go stimuli during active training. This correlation supports a functional role for left parietal structures in the plasticity of inhibitory control to NoGo stimuli (at least in the case of the present paradigm). No AEP effects were observed with Go stimuli during active training.

The specificity of the AEP effects, i.e., that only NoGo and not Go trials were affected, and their correlation with RT facilitation are not readily explained in terms of simple motor or procedural learning. If either of these were the case, AEPs from Go trials would likely have modulated, too. Plus, the fact that source activity significantly decreased argues against a straightfor- ward explanation in terms of enhanced attention to one type of stimulus or one region of space, both of which would be predicted to enhance responses after training. Instead, our pattern of results is consistent with low-level inhibitory control over prepotent responses to NoGo stimuli (although another potentially concurrent possibility is that these effects reflect changes in decision criterion).

Both the main effect of section and also the evidence for frontal activity that was modulated as a function of stimulus would suggest that top-down control processes are likely playing a more general role during active training. Still, additional experiments akin to those by Schröger (1993) would be required to better disentangle effects of spatial processing from response mapping in the present study.

The latency of our effect (i.e., 61-104 ms) suggests that training-induced plasticity in inhibitory control occurs during relatively early sensory processing stages. In this regard, it is worthwhile to situate the timing of the present effects with respect to general auditory processing as well as to spatial processing (Murray and Spierer, 2009; Spierer et al., 2010). Primary auditory cortices respond to sounds at $\sim 15 \mathrm{~ms}$ 
(Liégeois-Chauvel et al., 1994) with propagation along the superior temporal cortex within the subsequent 3-10 ms (Howard et al., 2000). Likewise, auditory-driven responses in frontal (Liasis et al., 2001) and even occipital (Romei et al., 2007, 2009) regions have been observed within the initial $30-60 \mathrm{~ms}$ after stimulus onset. Thus, although effects over the 61-104 ms poststimulus interval are indeed earlier than reported previously, they are unlikely reflecting exclusively feedforward or stimulus-driven activity, particularly in the temporoparietal regions identified by our source estimations (Inui et al., 2006).

The present findings over the 61-104 ms poststimulus interval are substantially earlier than what has been typically reported. Previous studies suggest that inhibition of prepotent responses to NoGo stimuli relies on top-down control involving frontoparietal networks over the 150-400 ms poststimulus onset interval (Schröger, 1993; Botvinick et al., 2001; Rubia et al., 2001; Aron et al., 2004; Polich, 2007). We therefore hypothesize that our effects during active training reflect the development of a low-level form of inhibition, mediating Go/NoGo performance improvement that may act in concert with top-down, consciously controlled, inhibitory processes. Accordingly, Verbruggen and Logan (2008) advanced that decreases in response time induced by Go/NoGo practice relies on the emergence of fast bottom-up suppression signals mediated by an increase in the strength of association or mapping between the stimulus and stop response (Shiffrin and Schneider, 1977; Logan, 1988). The parietal localization of the present source estimations provides an additional line of support to the proposition that inhibitory control develops based on the recurrence of consistent mappings of stimuli onto motor response withholding rules (Shiffrin and Schneider, 1977; Logan, 1988). Parietal structures are suitable candidates for comprising learned associations between stimuli and behavioral responses. On the one hand, parietal structures have been implicated in the control of motor planning (Watanabe et al., 2002), in the coordinate transformations required to convert sensory signals into motor commands (Andersen et al., 1997), as well as in the preparation for movements (Deiber et al., 1991, 1996; Decety et al., 1992). On the other hand, previous studies demonstrated the involvement of left (contralateral) temporoparietal networks in the integration of spatial information differentiating the Go and NoGo stimuli in our study (Spierer et al., 2007, 2008). Together, the evidence for a role of parietal structures in both sensorimotor interactions and auditory spatial processing suggest that the effect of Go/NoGo training revealed in the present study might reflect the labeling of right-lateralized NoGo stimuli with signals yielding motor response inhibition. This hypothesis is further supported by the significant correlation between RTs to Go stimuli and the decrease in left parietal response strength.

In contrast to the effect of training manifesting when participants were engaged in the active Go/NoGo task [i.e., modifications of responses to NoGo (right) but not Go stimuli], in the context of passive listening, the effect of training manifested as topographic modulations of AEPs to left (Go) but not right (NoGo) stimuli over the 31-81 ms interval. Source estimations performed over this period revealed a decrease in response strength to left stimuli (Go) within right temporal and anterior parietal areas.

Participants were trained to respond to left-lateralized stimuli, which presumably increased their behavioral relevance. We hypothesize that the training sessions modified responses to Go but not NoGo stimuli during the passive listening portion because, under passive listening (or other task-irrelevant) contexts, there is a functional advantage of nonetheless detecting stimuli that were recently behaviorally relevant. This would not be the case for behaviorally irrelevant (NoGo) stimuli. Supporting this hypothesis, our effect occurred at a latency and locus corresponding to the P50, a component associated with sensory gating mechanisms by which the auditory system prevents irrelevant and/or redundant sensory information from the environment accessing and overwhelming higher-order representations (Hsieh et al., 2004; Kisley et al., 2004; Lijffijt et al., 2009; Yadon et al., 2009). The decrease in response strength of P50 generators associated with learning to respond to Go stimuli may reflect facilitated access for these stimuli to reach higher processing stages. Several studies demonstrated associations between the strength of P50 and performance on attention or detection tasks (Wan et al., 2008; Thomas et al., 2010). P50 has also been shown to play a role in the maintaining of auditory attentive state (Wan et al., 2008). The findings during passive listening are consistent with the hypothesis that Go/NoGo training modified low-level stimulus representations.

Several putative mechanisms could account for suppressed responses to NoGo stimuli during active training. One possibility is that conflicts between Go and NoGo response requirements are reduced (Nieuwenhuis et al., 2003). Another possibility is that, during the course of training, brain responses become more selective or otherwise refined. Training has been proposed to result in the exclusion of irrelevant neural activity and thus in the increase in the selectivity and efficiency of neural activity (Schoups et al., 1998; Schiltz et al., 1999; Song et al., 2002; Kelly and Garavan, 2005). Accordingly, the recurrent mappings of NoGo stimuli onto inhibition rules could have reduced the activity involved in triggering motor responses to NoGo stimuli. Alternatively, recurrent inhibition of response to NoGo while executing speeded responses to Go stimuli could have modulated the attentional or cognitive demand required to apply the (NoGo) response rule to the NoGo stimuli (Hill and Schneider, 2006). With regard to evidence that unattended and task irrelevant stimuli are processed more slowly and less reliably in the brain (Kanwisher and Wojciulik, 2000; Kastner and Ungerleider, 2000; Lamme and Roelfsema, 2000), the decrease in response strength to NoGo stimuli could reflect training-induced lessening in the behavioral relevance attributed to right-lateralized stimuli, which in turn helped decrease response prepotency. In turn, during passive listening, this extended to effects on Go stimuli because of their high behavioral relevance during the task.

\section{References}

Andersen RA, Snyder LH, Bradley DC, Xing J (1997) Multimodal representation of space in the posterior parietal cortex and its use in planning movements. Annu Rev Neurosci 20:303-330.

Aron AR, Robbins TW, Poldrack RA (2004) Inhibition and the right inferior frontal cortex. Trends Cogn Sci 8:170-177.

Aron AR, Behrens TE, Smith S, Frank MJ, Poldrack RA (2007) Triangulating a cognitive control network using diffusion-weighted magnetic resonance imaging (MRI) and functional MRI. J Neurosci 27:3743-3752.

Blauert J (1997) Spatial hearing (revised edition). Cambridge, MA: Massachusetts Institute of Technology.

Botvinick MM, Braver TS, Barch DM, Carter CS, Cohen JD (2001) Conflict monitoring and cognitive control. Psychol Rev 108:624-652.

Decety J, Kawashima R, Gulyás B, Roland PE (1992) Preparation for reaching: a PET study of the participating structures in the human brain. Neuroreport 3:761-764.

Deiber MP, Passingham RE, Colebatch JG, Friston KJ, Nixon PD, Frackowiak 
RS (1991) Cortical areas and the selection of movement: a study with positron emission tomography. Exp Brain Res 84:393-402.

Deiber MP, Ibañez V, Sadato N, Hallett M (1996) Cerebral structures participating in motor preparation in humans: a positron emission tomography study. J Neurophysiol 75:233-247.

Dillon DG, Pizzagalli DA (2007) Inhibition of action, thought, and emotion: a selective neurobiological review. Appl Prev Psychol 12:99-114.

Eimer M (1993) Effects of attention and stimulus probability on ERPs in a Go/Nogo task. Biol Psychol 35:123-138.

Falkenstein M, Koshlykova NA, Kiroj VN, Hoormann J, Hohnsbein J (1995) Late ERP components in visual and auditory Go/Nogo tasks. Electroencephalogr Clin Neurophysiol 96:36-43.

Falkenstein M, Hoormann J, Hohnsbein J (1999) ERP components in Go/ Nogo tasks and their relation to inhibition. Acta Psychol (Amst) 101:267-291.

Falkenstein M, Hoormann J, Christ S, Hohnsbein J (2000) ERP components on reaction errors and their functional significance: a tutorial. Biol Psychol 51:87-107.

Grave de Peralta Menendez R, Gonzalez Andino S, Lantz G, Michel CM, Landis T (2001) Noninvasive localization of electromagnetic epileptic activity. I. Method descriptions and simulations. Brain Topogr 14:131-137.

Grave-de Peralta R, González-Andino S, Gómez-González CM (2004) The biophysical foundations of the localisation of encephalogram generators in the brain. The application of a distribution-type model to the localisation of epileptic foci. Rev Neurol 39:748-756.

Hill NM, Schneider W (2006) Brain changes in the development of expertise: neurological evidence on skill-based adaptations. In: Cambridge handbook of expertise and expert performance (Ericsson KA, Charness N, Feltovich P, Hoffman R, eds), pp 653-682. New York: Cambridge UP.

Howard MA, Volkov IO, Mirsky R, Garell PC, Noh MD, Granner M, Damasio H, Steinschneider M, Reale RA, Hind JE, Brugge JF (2000) Auditory cortex on the human posterior superior temporal gyrus. J Comp Neurol 416:79-92.

Hsieh MH, Liu K, Liu SK, Chiu MJ, Hwu HG, Chen AC (2004) Memory impairment and auditory evoked potential gating deficit in schizophrenia. Psychiatry Res 130:161-169.

Inui K, Okamoto H, Miki K, Gunji A, Kakigi R (2006) Serial and parallel processing in the human auditory cortex: a magnetoencephalographic study. Cereb Cortex 16:18-30.

Jodo E, Kayama Y (1992) Relation of a negative ERP component to response inhibition in a Go/No-go task. Electroencephalogr Clin Neurophysiol 82:477-482.

Kaiser S, Weiss O, Hill H, Markela-Lerenc J, Kiefer M, Weisbrod M (2006) $\mathrm{N} 2$ event-related potential correlates of response inhibition in an auditory Go/Nogo task. Int J Psychophysiol 61:279-282.

Kanwisher N, Wojciulik E (2000) Visual attention: insights from brain imaging. Nat Rev Neurosci 1:91-100.

Kastner S, Ungerleider LG (2000) Mechanisms of visual attention in the human cortex. Annu Rev Neurosci 23:315-341.

Kelly AM, Garavan H (2005) Human functional neuroimaging of brain changes associated with practice. Cereb Cortex 15:1089-1102.

Kiefer M, Marzinzik F, Weisbrod M, Scherg M, Spitzer M (1998) The time course of brain activations during response inhibition: evidence from event-related potentials in a go/no go task. Neuroreport 9:765-770.

Kisley MA, Noecker TL, Guinther PM (2004) Comparison of sensory gating to mismatch negativity and self-reported perceptual phenomena in healthy adults. Psychophysiology 41:604-612.

Lamme VA, Roelfsema PR (2000) The distinct modes of vision offered by feedforward and recurrent processing. Trends Neurosci 23:571-579.

Liasis A, Towell A, Alho K, Boyd S (2001) Intracranial identification of an electric frontal-cortex response to auditory stimulus change: a case study. Brain Res Cogn Brain Res 11:227-233.

Liégeois-Chauvel C, Musolino A, Badier JM, Marquis P, Chauvel P (1994) Evoked potentials recorded from the auditory cortex in man: evaluation and topography of the middle latency components. Electroencephalogr Clin Neurophysiol 92:204-214.

Lijffijt M, Lane SD, Meier SL, Boutros NN, Burroughs S, Steinberg JL, Gerard Moeller F, Swann AC (2009) P50, N100, and P200 sensory gating: relationships with behavioral inhibition, attention, and working memory. Psychophysiology 46:1059-1068.
Logan GD (1988) Automaticity, resources, and memory: theoretical controversies and practical implications. Hum Factors 30:583-598.

Michel CM, Murray MM, Lantz G, Gonzalez S, Spinelli L, Grave de Peralta R (2004) EEG source imaging. Clin Neurophysiol 115:2195-2222.

Murray MM, Spierer L (2009) Auditory spatio-temporal brain dynamics and their consequences for multisensory interactions in humans. Hear Res 258:121-133.

Murray MM, Brunet D, Michel CM (2008) Topographic ERP analyses: a step-by-step tutorial review. Brain Topogr 20:249-264.

Nieuwenhuis S, Yeung N, van den Wildenberg W, Ridderinkhof KR (2003) Electrophysiological correlates of anterior cingulate function in a go/ no-go task: effects of response conflict and trial type frequency. Cogn Affect Behav Neurosci 3:17-26.

Nieuwenhuis S, Yeung N, Cohen JD (2004) Stimulus modality, perceptual overlap, and the go/no-go N2. Psychophysiology 41:157-160.

Oldfield RC (1971) The assessment and analysis of handedness: the Edinburgh inventory. Neuropsychologia 9:97-113.

Pascual-Marqui RD, Michel CM, Lehmann D (1995) Segmentation of brain electrical activity into microstates: model estimation and validation. IEEE Trans Biomed Eng 42:658-665.

Perrin F, Bertrand O, Pernier J (1987) Scalp current density mapping: value and estimation from potential data. IEEE Trans Biomed Eng 34:283-288.

Pfefferbaum A, Ford JM, Weller BJ, Kopell BS (1985) ERPs to response production and inhibition. Electroencephalogr Clin Neurophysiol 60:423-434.

Polich J (2007) Updating P300: an integrative theory of P3a and P3b. Clin Neurophysiol 118:2128-2148.

Proctor RW, Shao C (2010) Does the contribution of stimulus-hand correspondence to the auditory Simon effect increase with practice? Exp Brain Res 204:131-137.

Romei V, Murray MM, Merabet LB, Thut G (2007) Occipital transcranial magnetic stimulation has opposing effects on visual and auditory stimulus detection: implications for multisensory interactions. J Neurosci 27:11465-11472.

Romei V, Murray MM, Cappe C, Thut G (2009) Preperceptual and stimulus-selective enhancement of low-level human visual cortex excitability by sounds. Curr Biol 19:1799-1805.

Rubia K, Russell T, Overmeyer S, Brammer MJ, Bullmore ET, Sharma T, Simmons A, Williams SC, Giampietro V, Andrew CM, Taylor E (2001) Mapping motor inhibition: conjunctive brain activations across different versions of go/no-go and stop tasks. Neuroimage 13:250-261.

Schapkin SA, Falkenstein M, Marks A, Griefahn B (2007) Practice-related effects in a Go-Nogo task. Percept Mot Skills 105:1275-1288.

Schiltz C, Bodart JM, Dubois S, Dejardin S, Michel C, Roucoux A, Crommelinck M, Orban GA (1999) Neuronal mechanisms of perceptual learning: changes in human brain activity with training in orientation discrimination. Neuroimage 9:46-62.

Schoups AA, Vogels R, Orban GA (1998) Effects of perceptual learning in orientation discrimination on orientation coding in V1. Invest Ophthalmol Vis Sci 39:s684.

Schröger E (1993) Event-related potentials to auditory stimuli following transient shifts of spatial attention in a Go/Nogo task. Biol Psychol 36:183-207.

Schröger E, Wolff C (1996) Mismatch response of the human brain to changes in sound location. Neuroreport 7:3005-3008.

Shiffrin RM, Schneider W (1977) Controlled and automatic human information processing. II. Perceptual learning, automatic attending, and a general theory. Psychol Rev 84:127-190.

Song Y, Ding Y, Fan S, Chen L (2002) An event-related potential study on visual perceptual learning under short-term and long-term training conditions. Neuroreport 13:2053-2057.

Spierer L, Tardif E, Sperdin H, Murray MM, Clarke S (2007) Learninginduced plasticity in auditory spatial representations revealed by electrical neuroimaging. J Neurosci 27:5474-5483.

Spierer L, Murray MM, Tardif E, Clarke S (2008) The path to success in auditory spatial discrimination: electrical neuroimaging responses within the supratemporal plane predict performance outcome. Neuroimage 41:493-503.

Spierer L, De Lucia M, Bernasconi F, Bourquin N, Clarke S, Murray MM (2010) Learning-induced plasticity in human audition: objects, time, and space. Hear Res. Advance online publication. Retrieved September 2, 2010. doi:10.1016/j.heares.2010.03.086 
Thomas C, vom Berg I, Rupp A, Seidl U, Schröder J, Roesch-Ely D, Kreisel SH, Mundt C, Weisbrod M (2010) P50 gating deficit in Alzheimer dementia correlates to frontal neuropsychological function. Neurobiol Aging $31: 416-424$

Thorpe SJ, Fabre-Thorpe M (2001) Neuroscience. Seeking categories in the brain. Science 291:260-263.

Tibshirani R, Walther G, Botstein D, Brown P (2005) Cluster validation by prediction strength. J Comput Graph Stat 14:511-528.

Verbruggen F, Logan GD (2008) Automatic and controlled response inhibition: associative learning in the go/no-go and stop-signal paradigms. J Exp Psychol Gen 137:649-672.

Vocat R, Pourtois G, Vuilleumier P (2008) Unavoidable errors: a spatiotemporal analysis of time-course and neural sources of evoked potentials associated with error processing in a speeded task. Neuropsychologia 46:2545-2555.

Walther S, Goya-Maldonado R, Stippich C, Weisbrod M, Kaiser S (2010) A supramodal network for response inhibition. Neuroreport 21:191-195.

Wan L, Friedman BH, Boutros NN, Crawford HJ (2008) P50 sensory gating and attentional performance. Int J Psychophysiol 67:91-100.

Watanabe T, Náñez JE Sr, Koyama S, Mukai I, Liederman J, Sasaki Y (2002) Greater plasticity in lower-level than higher-level visual motion processing in a passive perceptual learning task. Nat Neurosci 5:1003-1009.

Yadon CA, Bugg JM, Kisley MA, Davalos DB (2009) P50 sensory gating is related to performance on select tasks of cognitive inhibition. Cogn Affect Behav Neurosci 9:448-458. 